\title{
Faculty welcomes report on care homes
}

The Faculty of General Dental Practice (FGDP[UK]) has welcomed a new report by the Care Quality Commission (CQC) which highlights the poor provision of oral healthcare in residential care homes.

Around 400,000 people in the UK live in care homes, and a NICE Quality Standard ${ }^{1}$ published in 2017 recommends that residents are supported to clean their teeth and/or dentures daily, and have their oral health needs assessed on admission and recorded in their personal care plans.

However, the health inspectorate's 'Smiling matters'2 study of 100 care homes finds that one in six do not assess residents' oral health on admission, $52 \%$ do not have an oral health plan for residents, and $47 \%$ of staff had not received training in oral healthcare. Care homes specialising in dementia, which affects up to $70 \%$ of care home residents, were even less likely to have considered oral health needs. A third of homes also said they could not always access dental care for their residents.

Dr Paul Batchelor, editor of FGDP(UK)'s Dementia-friendly dentistry ${ }^{3}$ guidance (pictured), was a member of the NICE advisory committee which developed the quality standard, and is the Faculty's representative on the CQC advisory group on oral health in care homes. Commenting on the publication of Smiling Matters, he said:

'The Faculty has been working since 2013 to support improvement in oral healthcare arrangements for older members in society. We welcome the CQC's publication, which reinforces the need for improvement by care home providers, while recognising the wider determinants and highlighting the need for the health and care sectors to work together.

'We should also remember that the majority of those with dementia, almost 500,000 people, are living in the community rather than in residential care or nursing homes. GDPs and practice teams may therefore wish to consult our guidance on dementia-friendly dentistry, which is available free of charge, and enables them to understand dementia and its implications for dental practice, patient management and clinical decision-making.'

\section{References}

1. NICE. Oral health in care homes. Quality standard [QS151]. June 2017. Available at: https://www.nice.org.uk/guidance/QS151 (accessed July 2019).

2. Care Quality Commission. Smiling matters: oral health care in care homes. Last updated 25 June 2019. Available at: https://www.cqc.org.uk/publications/major-report/smilingmatters-oral-health-care-care-homes (accessed July 2019).

3. FGDP (UK). Dementia-friendly dentistry: good practice guidelines. 2017. Available at: https://www.fgdp.org.uk/guidance-standards/dementia-friendly-dentistry (accessed July 2019).

\section{Becoming a reflective practitioner}

The General Dental Council (GDC), along with eight other healthcare regulators, has published a joint statement on the importance and benefits of being a reflective practitioner.

The regulators have worked together to develop the statement, which sets out each of the organisations' requirements for reflection. It also offers advice for practitioners on how to approach reflection effectively, both as individuals and as part of teams.

The work is part of a shared commitment to drive an open and honest culture where professionals feel able to reflect on their experiences to support development and learning.

As well as reinforcing how reflection can help dental professionals gain insight into their whole practice, the statement highlights the direct impact it can have on improving services and patient care.

It endorses the value of dental practitioners reflecting in groups, teams and multi-professional settings to help develop ideas that can bring about positive change in practice. As part of this, it makes it clear that employers should encourage their teams to make time for reflection as a way of aiding development, improving wellbeing and deepening professional commitment.

The full statement can be accessed from https://www. gdc-uk.org/professionals/cpd/reflective-practice. 